\title{
Epicardial adipose tissue thickness assessed by cardiac magnetic resonance is an independent indicator for coronary artery stenosis in asymptomatic type 2 diabetic patients
}

Hee Yeong Kim*, Young Jin Kim, Byoung Wook Choi

From 15th Annual SCMR Scientific Sessions

Orlando, FL, USA. 2-5 February 2012

\section{Background}

We investigated the association between epicardial adipose tissue (EAT) thickness and myocardial ischemia as well as coronary artery stenosis assessed by cardiovascular magnetic resonance (CMR) in asymptomatic type 2 diabetic patients.

\section{Methods}

A total of 100 type 2 diabetic subjects (51 men and 49 women; mean age: $56.4 \pm 7.6$ years) were enrolled. Silent myocardial ischemia by CMR was defined as an evidence of inducible ischemia or myocardial infarction and signal reduction or stenosis of $\geq 50 \%$ in the vessel diameter were used as the criteria for significant coronary artery stenosis on coronary MR angiography.

\section{Results}

EAT thickness was positively correlated with BMI, waist-to-hip ratio, systolic blood pressure, postprandial glucose, fasting/postprandial triglyceride, HbA1c level, and HOMA-IR. A total of 24 patients had significant coronary artery stenosis and 14 patients had silent myocardia ischemia in CMR (3 with silent myocardial infarction, 13 with inducible ischemia, 2 with both). EAT thickness was higher in patients who had significant stenosis; however, it was not different between the subjects with silent myocardial ischemia and the subjects with no evidence of silent myocardial ischemia in CMR (13.0 $\pm 2.6 \mathrm{~mm}$ vs. $11.5 \pm 2.1 \mathrm{~mm}, \mathrm{p}=0.01,12.8 \pm 2.1$ vs. 11.7 $\pm 2.3 \mathrm{~mm}, \mathrm{p}=0.11$, respectively). In multivariate logistic

regression analysis, EAT thickness was an independent indicator for significant coronary artery stenosis after adjusting for traditional risk factors (OR 1.353, $\mathrm{p}=0.031$ ).

\section{Conclusions}

Increased EAT thickness assessed by CMR is an independent risk factor for significant coronary artery stenosis in asymptomatic type 2 diabetes; however, the thickness was not associated with silent myocardial ischemia.

\section{Funding}

None.

Published: 1 February 2012

doi:10.1186/1532-429X-14-S1-P78

Cite this article as: Kim et al:: Epicardial adipose tissue thickness assessed by cardiac magnetic resonance is an independent indicator for coronary artery stenosis in asymptomatic type 2 diabetic patients. Journal of Cardiovascular Magnetic Resonance 2012 14(Suppl 1):P78. 